\title{
A Multi-Antenna Based Spectrum Sharing for IMT-Advanced SDMA Systems
}

\author{
Han-Shin Jo \\ Dept. Of Electronics and Control Engineering, Hanbat National University \\ Daejeon, Korea \\ hsjo@hanbat.ac.kr
}

\begin{abstract}
We propose a simple multi-user beamforming to assist space-division multiple access (SDMA) as well as to mitigate interference from IMT-Advanced base station (BS) to fixedsatellite service (FSS) earth stations (ESs). The BS with multiple antennas forms multiple beams that steer nulls, i.e., emit quite low power (interference) to the directions of the ESs. The beamforming vectors are designed by inverting the array steering vectors corresponding to the direction angles of the ESs as well as random direction angles of the main beam lobes. In order to show the merits of the proposed method, we model interference power at the ES and compute the minimum separation distances between the BS and the ESs required for their coexistence. Even for the case of the multiple ESs, the proposed method can reduce the minimum separation distance by up to 92 percent without any expense of additional radio spectrum resources.
\end{abstract}

Keywords: Asymptotic throughput scaling, space-division multiple access (SDMA), spectrum sharing, precoding, feedback overhead, null-steering

\section{Introduction}

International telecommunication union for radiocommunication (ITU-R) working party (WP) 8F had done preparatory work for discovering new frequency band for IMT-Advanced systems, where 3.4-4.2 GHz band is determined as one of candidate bands [1]. To confirm the band usage, we need a feasibility study on coexistence (spectrum sharing) between IMTAdvanced system and fixed-satellite services (FSS) currently being in the band. It is shown that the two systems should be at least about $40 \mathrm{~km}$ far from each other for their coexistence [1], however the separation distance is too large to allow for practical IMT-Advanced service. In $[2,3,4,5,6]$, null steering beamforming is proposed to mitigate the interference from IMTAdvanced base station (BS), which dramatically reduces the minimum separation distance required for spectrum sharing with FSS. However, the method focuses on interference mitigation to just one FSS earth station (ES) and is not effective to mitigating interferences to the multiple FSS ESs. It is desirable to develop advanced interference mitigation for spectrum sharing between IMT-Advanced BS and multiple ESs.

In broadcast multi-user multiple-input multiple-output (MIMO) channels, simultaneous transmission to multiple users, known as space division multiple access (SDMA), can achieve very higher throughput. Although dirty paper coding (DPC) can achieve the sum capacity [7], its encoding and decoding complexity motives practical SDMA algorithms such as zeroforcing beamforming (ZFBF) [8][9]. 


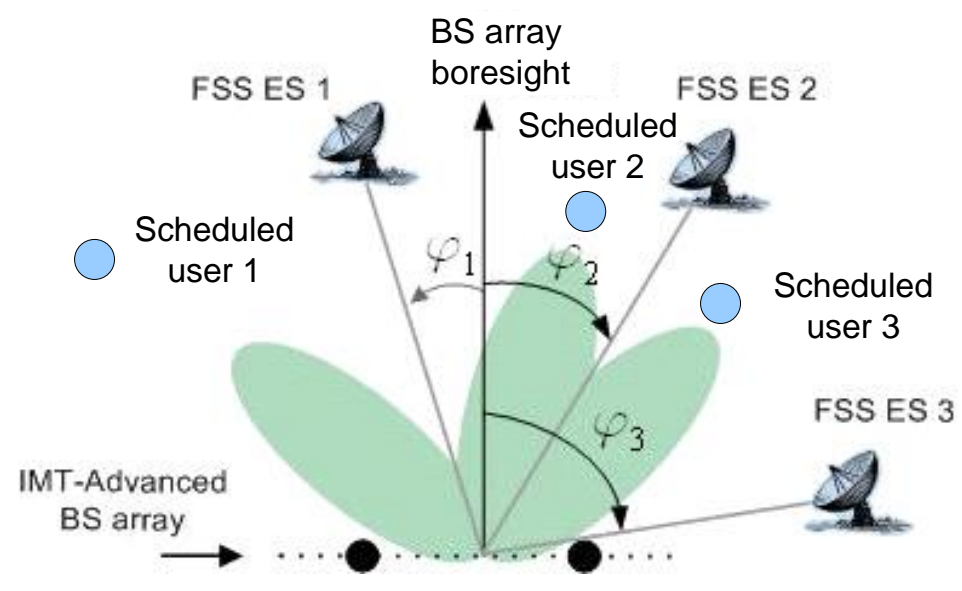

\section{Figure 1. Interference Scenario of IMT-Advanced BS with the Proposed Interference Mitigation Algorithm}

In $[8,9]$, in order to avoid interference among user streams, beamforming matrix can be calculated from the channel transfer matrix when assuming the perfect channel state information (CSI) at BS. CSI is usually obtained at the mobile users linked BS, and sent to the BS over a limited-rate feedback channel. However, these schemes cannot be employed to mitigate interferences from IMT-Advanced BS to FSS ES, because the BS cannot utilize the CSI between the ES and the BS (e.g., the ES can't estimate CSI from the IMT-Advanced systems).

To overcome the above mentioned drawbacks of CSI-based beamformings, we proposed the multiuser beamforming scheme based on the information of the direction angles of FSS ESs (AOEs) and the direction angles of main beam lobes (AOBs). First, AOE information can be obtained from a database storing two-dimensional coordinates of FSS ESs and IMTAdvanced BSs. Second, each AOB is determined by generating uniform random variable which is distributed over but not equal to the AOE. Third, beamforming vectors can be easily founded by inverting the array steering vectors corresponding to the AOE and the AOB, so that the transmit array constructs main beams in AOB for a transmit target user; moreover, transmit array constructs spatial nulls in AOB of other users and AOE.

\section{System Model}

Before the system model descriptions, we define notations used throughout this paper. $\mathbf{A}^{\mathrm{T}}$ represents the transpose of a matrix $\mathbf{A}, \mathbf{A}^{-1}$ the inverse of a matrix $\mathbf{A}, \mathbf{I}_{R}$ an $R \times R$ identity matrix, and $[\mathbf{A}]_{r, t}$ the element in the $r$ th row and $t$ th column of a matrix A . Moreover, $\mathbf{0}_{r, t}$ and $\mathbf{1}_{R, D}$ mean a $r \times t$ zero matrix and a $R \times 1$ column vector, respectively. The column vector $\mathbf{1}_{R, D}$ is defined in (1).

$$
\left[\mathbf{1}_{R, D}\right]_{i, 1}= \begin{cases}1 & \text { if } i=D \\ 0 & \text { if } i \neq D\end{cases}
$$


Consider a MIMO downlink of IMT-Advanced system, where a BS with $N$ antennas sends data to $K$ users with $M$ antennas through a frequency-flat block fading channel. $\mathbf{y}_{k}$ is a $M \times 1$ vector of received signals of the $k$ th user expressed as

$$
\mathbf{y}_{k}=\sqrt{\frac{\bar{\gamma}_{k}}{N}} \mathbf{H}_{k} \mathbf{x}+\mathbf{w}_{k},
$$

where $\mathbf{x}$ is a $N \times 1$ vector of transmitted signals, $\bar{\gamma}_{k}$ is average signal-to-noise ratio (SNR) per receiving antenna, $\mathbf{H}_{k}$ is an $M \times N$ complex channel matrix whose entries are spatially correlated zero-mean complex Gaussian. $\mathbf{w}_{k}$ is an $M \times 1$ zero-mean spatially-white complex Gaussian receiver noise vector of covariance matrix $N_{0} \mathbf{I}_{M}$. Moreover, $P(\leq N-1)$ FSS ESs sharing same frequency bands with IMT-Advanced are considered as shown in Fig. 1.

We assume SDMA that constructs $L(=N-P)$ beams and transmits data to users $\left\{k_{l}^{*}\right\}_{l=1, \cdots, L}$ each with the highest SINR via the beamforming vectors $\left\{\mathbf{w}_{l}\right\}_{l=1, \cdots, L}$ as follows.

$$
k_{l}^{*}=\arg \max _{k \in\{1,2, \cdots, K\}} \gamma_{k, l}, \quad l=1, \cdots, L
$$

The transmitted signal is then

$$
\mathbf{x}=\mathbf{W} \mathbf{s}=\sum_{l=1}^{L} \mathbf{w}_{l} s_{l},
$$

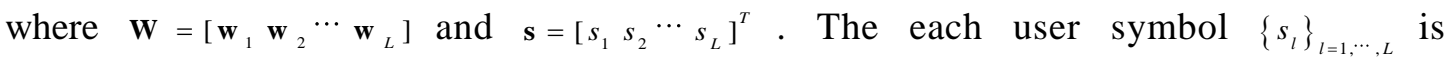
recovered at the corresponding users with a minimum mean square error (MMSE) detection. The SINR $\gamma_{k, l}$ on the $l$ th stream weighted by $\mathbf{w}$ of the $k$ th receiver is given by

$$
\gamma_{k, l}(\mathbf{W})=\frac{1}{\left\lceil\left(\frac{\bar{\gamma}_{k}}{L} \tilde{\mathbf{H}}_{k}^{H} \tilde{\mathbf{H}}_{k}+\mathbf{I}_{L}\right)^{-1}\right\rfloor_{l, l}}-1,
$$

where $\tilde{\mathbf{H}}_{k}=\mathbf{H}_{k} \mathbf{W}$.

\section{Interference Mitigation Algorithm}

As the frequency bands of the 3.4-4.2 GHz considered in this paper are only allocated downlink (space to earth) FSS, there are no interferences from FSS ES to IMT-Advanced system in target frequency bands. In case of interference from geosynchronous orbit satellite to IMT-Advanced, there is no interesting issue since the interference is sufficiently weak due 
to low transmit power of a satellite and large propagation loss in the link between the satellite and IMT-Advanced system on earth. Therefore, we focus on the interference from IMTAdvanced BS to FSS ES as shown Figure 1. Figure 1 shows that IMT-Advanced BS constructs nulls at $\operatorname{AOE} \varphi_{1}, \varphi_{2}$, and $\varphi_{3}$ by using the proposed interference mitigation technique.

The main part of the proposed inference mitigation is a construction of beamformig vectors, each of which forms $P$ nulls (zero level of beam pattern) at the AOEs and a main beam (peak level of beam pattern) at a certain AOB. The beamforming vectors can be easily computed by inverting the array steering vectors corresponding to the AOEs and the AOBs. Due to a "fixed" nature of FSS-ES and IMT-Advanced BS, AOE information can be calculated from the database of the FSS ES and IMT-Advanced BS position such as longitude and latitude. We assume that AOE information is already known to IMT-Advanced BS. AOBs, $\left\{\theta_{i}\right\}_{i=1, \cdots, L}$ are random variables uniformly distributed over $[-\pi / 2, \pi / 2]$ (which also are the directions of IMT-Advanced users selected in (3)), but not equal to AOE, $\left\{\varphi_{i}\right\}_{i=1, \cdots, P}$, to suppress the interference power from IMT-Advanced BS to FSS ESs.

The beamforming vectors are designed to satisfy two conditions: 1) construction of main beam lobes in AOB for a transmit target user 2) construction of spatial nulls in AOB of other users and AOE. Therefore, the $l$ th beamforming vector $\left\{\mathbf{w}_{l}\right\}_{l=1, \cdots L}$ is given by the following set of equations:

$$
\begin{aligned}
& \mathbf{w}_{l}^{\mathrm{T}} \mathbf{a}\left(\varphi_{i}\right)=0, \quad(i=1,2, \cdots, P), \\
& \mathbf{w}_{l}^{\mathrm{T}} \mathbf{a}\left(\theta_{i}\right)=\left\{\begin{array}{ll}
1, & \text { if } l=i, \\
0, & \text { if } l \neq i,
\end{array} \quad(i=1,2, \cdots, L) .\right.
\end{aligned}
$$

From these equations, we obtain the following matrix equation.

$$
\mathbf{W}^{\mathrm{T}} \mathbf{A}=\mathbf{U}^{\mathrm{T}}
$$

where $\mathbf{W}=\left[\begin{array}{lll}\mathbf{w}_{1} & \cdots & \mathbf{w}_{L}\end{array}\right]$ is an $N \times L$ matrix. $\mathbf{A}=\left[\mathbf{a}\left(\theta_{1}\right) \cdots \mathbf{a}\left(\theta_{L}\right) \mathbf{a}\left(\varphi_{1}\right) \cdots \mathbf{a}\left(\varphi_{P}\right)\right],(L+P=N)$ is an $N \times N$ matrix and $\mathbf{U}=\left[\begin{array}{lll}\mathbf{1}_{N, 1} & \cdots & \mathbf{1}_{N, L}\end{array}\right]$ is an $N \times L$ matrix. From (7) we can finally evaluate the matrix of beamforming vectors as

$$
\mathbf{W}=\left(\mathbf{A}^{-1}\right)^{T} \mathbf{U}
$$

When a linear array of $N$ isotropic antenna elements with uniform spacing is considered, the array steering vector (or named steering vector) at a given angle $\theta$ with respect to the array boresight $\mathrm{a}(\theta)$ is defined by [10] 


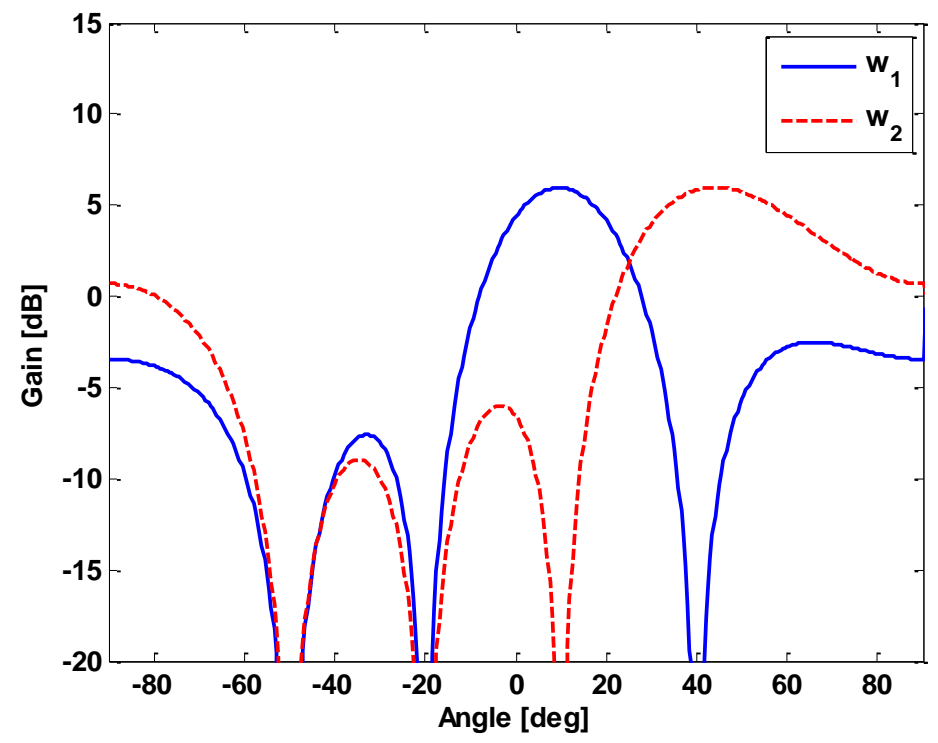

Figure 2. Beam Patterns Formed by Transmit Weight Vectors, $w_{1}$ and $\mathbf{w}_{2}$

$$
\mathbf{a}(\theta)=\left[\begin{array}{llll}
1 & e^{j 2 \pi \frac{w}{\lambda} \sin \theta} & \cdots & e^{j 2 \pi(N-1) \frac{w}{\lambda} \sin \theta}
\end{array}\right]^{T},
$$

where $w$ is an antenna spacing and $\lambda$ is wavelength of carrier signal.

Let us consider an example where there are two FSS ESs at $\varphi_{1}=-50^{\circ}$ and $\varphi_{2}=-20^{\circ}$. IMTAdvanced BS is equipped with four antennas with half wavelength spacing between the antennas $(d=0.5 \times \lambda)$. IMT-Advanced BS computes the beamforming matrix $\mathbf{W}$ as $(8)$, on the basis of a matrix of array response vectors, $\mathbf{A}=\left[\mathbf{a}\left(\theta_{1}=10^{\circ}\right) \mathbf{a}\left(\theta_{2}=40^{\circ}\right) \mathbf{a}\left(\varphi_{1}=-50^{\circ}\right) \mathbf{a}\left(\varphi_{2}=-20^{\circ}\right)\right]$ and a matrix $\mathbf{U}=\left[\begin{array}{lll}\mathbf{1}_{4,1} & \mathbf{1}_{4,2}\end{array}\right]$ where $\mathbf{1}_{4,1}=\left[\begin{array}{llll}1 & 0 & 0 & 0\end{array}\right]^{\mathrm{T}}$ and $\mathbf{1}_{4,2}=\left[\begin{array}{llll}0 & 1 & 0 & 0\end{array}\right]^{\mathrm{T}}$. The beam patterns of the example beamforming matrix are plotted in Fig. 2. We observe that the vectors $\mathbf{w}_{1}$ and $\mathbf{w}_{2}$ each construct main beams at the AOB corresponding to the target user. Moreover, they construct spatial nulls in AOB of other users and AOE. More concretely, $w_{1}$ constructs a main beam at AOB $\left(\theta_{1}=10^{\circ}\right)$ and three nulls at both AOEs $\left(\varphi_{1}=-50^{\circ}, \varphi_{2}=-20^{\circ}\right)$ and another target AOB $\left(\theta_{2}=40^{\circ}\right)$. The $\mathbf{w}_{2}$ forms a main beam at AOB $\left(\theta_{1}=40^{\circ}\right)$ and three nulls at both $\operatorname{AOEs}\left(\varphi_{1}=-50^{\circ}, \varphi_{2}=-20^{\circ}\right)$ and another target $\operatorname{AOB}\left(\theta_{1}=10^{\circ}\right)$.

The proposed method can mitigate the interference to multiple FSS ESs located at AOE, the number AOE is less than $N$. However, in some countries such as France, Sweden, Japan, Korea, there is a limited number (twenty or less) of registered FSS ESs. Furthermore, since the FSS ESs are sparsely located, a few FSS ESs only need to be protected against the interference from a certain IMT-Advanced BS.

\section{Interference Assessment and Minimum Separation Distance}

It is universally said that frequency sharing between wireless communications is possible, when they operate satisfying predefined threshold of performance such as data rate or bit (or 
frame) error rate in spite of the additional interference from other systems in co-channel or adjacent channel. Power level of interference at the antenna of a victim receiver is considered as an evaluation indicator in most frequency sharing study. More concretely, the studies define the permissible interference power for $p \%$ of the time, which means the maximum allowable interference power for all except $p \%$ of the time [11]. When $p$ is a small percentage of the time, in a range of $0.001 \%$ to $1.0 \%$, the interference is referred to as "short term", and it is referred to as "long term" for $p \geq 20 \%$.

Path loss model is essential for analytical prediction of the interference power at a victim receiver. In this paper we adopt ITU-R Recommendation P.452 model whose accuracy and reliability have been verified by many previous studies on the coexistence between FSS earth station and terrestrial wireless communication systems. In the model, path loss is given as

$$
L_{P}=32.5+20 \log _{10} d+20 \log _{10} f+L_{C}^{(T)}+L_{C}^{(R)},
$$

where $d$ is the propagation distance in kilometer $(\mathrm{km})$ between a transmitter and a receiver, and $f$ represents the carrier frequency in megahertz. $L_{C}^{(T)}$ and $L_{c}^{(R)}$ are clutter losses, which is the loss of filed strength caused by objects such as buildings or trees around transmitters and receivers, respectively (but not by terrain such as mountains or hills). Clutter loss is calculated as [12]

$$
L_{C}=10.25 \times e^{-d_{c}}\left(1-\tanh \left[6\left(\frac{h}{h_{c}}-0.625\right)\right] \mid\right]-0.33,
$$

where $h$ and $h_{c}$ are respectively the height of the antenna and the clutter in meter (m), and $d_{c}$ denotes the distance in $\mathrm{km}$ between a clutter and the antenna. Plugging $h_{,} h_{c}$, and $d_{c}$ for each transmitter and receiver into (11), we obtain $L_{c}^{(T)}$ and $L_{c}^{(R)}$. In P.452 model, $h_{c}$ and $d_{c}$ are defined for each clutter category; village center, suburban, dense urban, urban, and highrise urban. We consider suburban as the clutter category where $h_{c}=9 \mathrm{~m}$ and $d_{c}=0.025 \mathrm{~km}$.

We now compute the interference power at a victim FSS ES from IMT-Advanced BS as follows.

$$
I=T_{B S}+A_{B S}+A_{E S}-L_{P}+L_{r},
$$

where $A_{E S}$ is the ES antenna gain, $T_{B S}$ is the BS transmit power, and $A_{B S}$ is the BS power gain. The $A_{B S}$ is a sum of antenna gain $A_{A N}(\psi)$ and beamforming gain $A_{B F}(\psi)$, i.e.,

$$
A_{B S}=A_{A N}(\psi)+A_{B F}(\psi),
$$

where $\psi$ is the angle with respect to antenna boresight, and ${ }_{A_{A N}(\psi)}$ is given by [13]

$$
A_{A N}(\psi)=A_{\max }-\min \left(12\left(\frac{\psi}{\psi_{3 d B}}\right), A_{m}\right),-180^{\circ} \leq \psi \leq 180^{\circ}
$$


Here, $A_{\mathrm{max}}$ is the maximum antenna gain, $A_{m}=20 \mathrm{~dB}$ is the maximum attenuation, and $\psi_{3 A B}=70^{\circ}$ is the $3 \mathrm{~dB}$ beam width. The beamforming gain $A_{B F}(\psi)$ is given from the use of beamforming matrix $\mathbf{w}=\left[\begin{array}{lll}\mathbf{w}_{1} & \cdots & \mathbf{w}_{L}\end{array}\right]$ in (8), which is computed as

$$
A_{B F}(\psi)=20 \log _{10}\left|\frac{1}{L} \sum_{i=1}^{L} \mathbf{w}_{i}^{T} \mathbf{a}(\psi)\right|,
$$

where $\mathbf{a}(\psi)$ is the steering vector defined in (9), and $L_{r}$ denotes the interfering signal power loss which is the ratio (in $\mathrm{dB}$ ) of the interference power in the frequency band of a victim system to $T_{B S}$. Note that $L_{r}$ is always less than zero from the definition, whereas $L_{P}$ is always larger than zero. The $L_{r}$ is caused by frequency separation between the two systems and imperfect filtering in a transmitter (IMT-Advanced BS) and a receiver (FSS ES). When the BS using orthogonal frequency-division multiplexing (OFDM) with a rectangular pulse is considered, $L_{r}$ is given by [14].

$$
\begin{array}{r}
L_{r}=10 \log _{10}\left\lceil\frac { P _ { s } } { 2 \pi T _ { B S } } \sum _ { i = 0 } ^ { U - 1 } \left\lceil\frac{1}{\pi\left(R_{-}-i\right)}-\frac{1}{\pi\left(R_{+}-i\right)}-\frac{\cos 2 \pi\left(R_{-}-i\right)}{\pi\left(R_{-}-i\right)}+\frac{\cos 2 \pi\left(R_{+}-i\right)}{\pi\left(R_{+}-i\right)}\right.\right. \\
+2 \sum_{k=1}^{\infty} \frac{\left.\left.(-1)^{k-1}\left[\left(2 \pi\left(R_{+}-i\right)\right)^{2 k-1}-\left(2 \pi\left(R_{-}-i\right)\right)^{2 k-1}\right]\right\rceil\right]}{(2 k-1)(2 k-1) !}||,
\end{array}
$$

where

$$
R_{+}=\frac{f}{R_{s}}+\frac{W_{V}}{2 R_{s}} \text { and } \quad R_{-}=\frac{f}{R_{s}}-\frac{W_{V}}{2 R_{s}},
$$

and $U$ is the number of subcarriers and $P_{s}$ is the transmit power per subcarrier. $R_{s}$ is subcarrier spacing and $W_{v}$ is the bandwidth of a victim receiver. 
Table 1. Simulation Parameters

\begin{tabular}{|c|c|c|}
\hline System & Parameter & Value \\
\hline \multirow[t]{7}{*}{ FSS ES } & Center frequency $(\mathrm{GHz})$ & 4 \\
\hline & Bandwidth (MHz) & 72 \\
\hline & Maximum antenna gain $(\mathrm{dBi})$ & 41 \\
\hline & Antenna height $(\mathrm{m})$ & 5 \\
\hline & Antenna type & Dish(Diameter : $3.8 \mathrm{~m}$ ) \\
\hline & Antenna pattern & $\begin{array}{c}\text { the ratio between the } \\
\text { antenna diameter and the } \\
\text { wavelength is less than or } \\
\text { equal to } 100[15]\end{array}$ \\
\hline & $\begin{array}{l}\text { Maximum permissible } \\
\text { interference power } \\
\text { for } \mathrm{I} / \mathrm{N}=-12.2 \mathrm{~dB}[16] \\
(\mathrm{dBW} / 72 \mathrm{MHz})\end{array}$ & -142.23 \\
\hline \multirow{9}{*}{$\begin{array}{c}\text { IMT } \\
\text { Advanced } \\
\text { BS }\end{array}$} & $\begin{array}{l}\text { Center frequency of operation } \\
(\mathrm{GHz})\end{array}$ & 4 \\
\hline & Transmit power $(\mathrm{dBW})$ & 13 \\
\hline & Number of transmit antennas & 4,8 \\
\hline & Antenna pattern & $\begin{array}{c}\text { 3-sector pattern Eq.(13) and } \\
(14)\end{array}$ \\
\hline & Antenna height (m) & 15 \\
\hline & Bandwidth (MHz) & 10 \\
\hline & Subcarrier spacing $(\mathrm{KHz})$ & 9.76 \\
\hline & Number of subcarriers & 1024 \\
\hline & Maximum antenna gain $(\mathrm{dBi})$ & 12 \\
\hline
\end{tabular}

Spectrum sharing between IMT-Advanced BS and FSS ES is possible when the interference in (12) is less than or equal to the permissible interference power $I_{\max }$, from which the minimum path loss required for spectrum sharing can be defined as

$$
\begin{aligned}
L_{P, \min } & =32.5+20 \log _{10} d_{\min }+20 \log _{10} f+L_{C}^{(T)}+L_{C}^{(R)}, \\
& =T_{B S}+A_{B S}+A_{E S}+L_{r}-I_{\max }
\end{aligned}
$$

where $d_{\min }$ is the minimum separation distance defined as the distance yielding the minimum path loss. From (18), it is obtained by

$$
d_{\min }=10 \frac{\frac{T_{B S}+A_{B S}+A_{E S}+L_{r}-I_{\max }-32.5-20 \log _{10} f-L_{C}^{(T)}-L_{C}^{(R)}}{20}}{20} .
$$

This indicates that IMT-Advanced BS and FSS ES being at least $d_{d_{\min }}$ apart can share their spectrum with each other. 


\section{Numerical Results}

In order to verify the feasibility of our proposed method, sharing analyses are carried out in the two scenarios of $N=4, P=3$ and $N=8, P=6$. Simulation parameters used in calculation are listed in Table 1.

Table 2. Minimum Separation Distances

(a) Scenario 1

\begin{tabular}{|l|c|c|}
\hline \multirow{2}{*}{$\begin{array}{l}\text { Scenario } 1 \\
(N=4, P=3)\end{array}$} & \multicolumn{2}{|c|}{ Minimum separation distance $(\mathrm{km})$} \\
\cline { 2 - 3 } & $\begin{array}{c}\text { interference } \\
\text { mitigation }\end{array}$ & $\begin{array}{c}\text { Without } \\
\text { interference } \\
\text { mitigation }\end{array}$ \\
\hline ES 1 (DOE: $\left.-50^{\circ}\right)$ & 1.4 & 28 \\
\hline ES 2 (DOE: $\left.-20^{\circ}\right)$ & 3.5 & 44 \\
\hline ES 3 (DOE: $\left.40^{\circ}\right)$ & 3.3 & 37.5 \\
\hline
\end{tabular}

(b) Scenario 2

\begin{tabular}{|c|c|c|}
\hline \multirow{2}{*}{$\begin{array}{l}\text { Scenario } 2 \\
(N=8, P=6)\end{array}$} & \multicolumn{2}{|c|}{ Minimum separation distance $(\mathrm{km})$} \\
\hline & $\begin{array}{l}\text { interference } \\
\text { mitigation }\end{array}$ & $\begin{array}{c}\text { Without } \\
\text { interference } \\
\text { mitigation }\end{array}$ \\
\hline ES 1 (DOE: $\left.-60^{\circ}\right)$ & 0.7 & 20 \\
\hline ES 2 (DOE: $\left.-45^{\circ}\right)$ & 2.4 & 30 \\
\hline ES 3 (DOE: $\left.-10^{\circ}\right)$ & 14 & 47.5 \\
\hline ES 4 (DOE: $\left.40^{\circ}\right)$ & 0.85 & 37.5 \\
\hline ES 5 (DOE: $\left.50^{\circ}\right)$ & 0.05 & 28 \\
\hline ES 6 (DOE: $\left.55^{\circ}\right)$ & 0.08 & 25 \\
\hline
\end{tabular}

The FSS ES with a bandwidth of $72 \mathrm{MHz}$ operates at a center frequency of $4 \mathrm{GHz}$. The permissible interference power $I_{\max }$ is $-142.23 \mathrm{dBW} / 72 \mathrm{MHz}$, which is calculated from the long term interference criterion $I / N=-12.2 \mathrm{~dB}$ given in [16], where $N$ is thermal noise power. We assume the $5^{\circ} \mathrm{FSS}$ ES elevation angles which cover the worst case requiring the largest minimum separation distance.

Table 2 lists the minimum separation distances between IMT-Advanced BS with the proposed interference mitigation technique and FSS ES. The all required minimum distances are smaller than $3.5 \mathrm{~km}$ compared with $44 \mathrm{~km}$ without mitigation technique. Although minimum separation distance of $3.5 \mathrm{~km}$ is obtained with help of the proposed interference mitigation technique, complete frequency sharing between two systems is not provided. In order to accomplish complete frequency sharing, the IMT-Advanced BS located in the area less than $3.5 \mathrm{~km}$ from the FSS ES must utilize other frequency bands. Nevertheless, the increasing demands for wireless services have induced recent radio spectrum shortages. Given that spectrum is limited and is a scare natural source, it should be used in such a way to increase spectrum efficiency. Not providing complete solution for coexistence problem, the proposed interference mitigation scheme can reduce the minimum separation distance up to $92 \%(44 \mathrm{~km} \rightarrow 3.5 \mathrm{~km})$ without any expense of additional radio spectrum resources. 


\section{Conclusions}

We have developed a simple and efficient interference mitigation algorithm using multiple null steering beamforming for SDMA as well as for frequency sharing between IMTAdvanced BS and multiple FSS ESs. The beamforming vectors generate power nulls to the direction angles of FSS ESs and peak power to the direction angles of the scheduled IMTAdvanced users. We mainly observe that the minimum separation distances can be reduced up to $92 \%$ without the expense of additional radio spectrum resources.

\section{Acknowledgement}

This research was supported by the MSIP(Ministry of Science, ICT and Future Planning), Korea, under the Global IT Talent support program (NIPA-2014ITAH0904140110020001000100100) supervised by the NIPA(National IT Industry Promotion Agency), and also it was supported by the Basic Science Research Program through the National Research Foundation of Korea (NRF) funded by the Ministry of Education, Science and Technology (2013R1A1A1005731).

\section{References}

[1] ITU-R Report M.2109, Sharing studies between IMT Advanced systems and geostationary satellite networks in the fixed-satellite service in the $3400-4200$ and 4 500-4 $800 \mathrm{MHz}$ frequency bands, (2007).

[2] J. Lim, H. S. Jo, H. G. Yoon and J. G. Yook, "Interference Mitigation Technique for the Sharing between IMT-Advanced and Fixed Satellite Service", Journal of Communications and Networks, vol. 9, no. 2, (2007).

[3] H. S. Jo, "Codebook-based precoding for SDMA-OFDMA with spectrum sharing", ETRI Journal, vol. 33, no. 6, (2011), pp. 831-840.

[4] H. S. Jo, "Spectrum Sharing SDMA with Limited Feedback: Throughput Analysis", KSII Trans. on Internet and Information Systems, vol. 6, no. 12, (2012), pp. 3237-3256.

[5] C. Mun and H. S. Jo, "Throughput Analysis of Transmit-Nulling SDMA with Limited Feedback", EURASIP Journal on Wireless Communications and Networking, (2013).

[6] H. S. Jo and C. Mun, "Transmit-nulling SDMA for coexistence with fixed wireless service", Journal of Electromagnetic Engineering and Science, vol. 11, (2011), pp. 34-41.

[7] H. Weingarten, Y. Steinberg and S. Shamai (Shitz), "The Capacity Region of the Gaussian MIMO Broadcast channel", Proc. IEEE International Symposium on Information Theory, (2004).

[8] G. Caire and S. Shamai, "On the achievable throughput of multiantenna Gaussian broadcast channel", IEEE Trans. Inform. Theory, vol. 49, no. 7, (2003).

[9] T. Yoo and A. Goldsmith, "Optimality of zero-forcing beam forming with multiuser diversity", Proceedings of the ICC Wireless Communications Theory, (2005).

[10] L. C. Godara, "Application of antenna arrays to mobile communications II. Beam-forming and direction-of-arrival considerations", Proceedings of the IEEE, vol. 85, no. 8, (1997).

[11] ITU-R Recommendation SF.1006, Determination of the interference potential between earth stations of the fixed-satellite service and stations in the fixed service, (1993).

[12] ITU-R Recommendation P.452-8, Prediction procedure for the evaluation of microwave interference between stations on the surface of the earth at frequency above about $0.7 \mathrm{GHz},(\mathbf{1 9 9 7})$.

[13] 3GPP2/TSG-C.R1002, 1xEV-DV evaluation methodology (V12.1), NOKIA, (2003).

[14] H. S. Jo, H. G. Yoon, J. Lim, W. G. Chung, J. G. Yook and H. K. Park, "The Coexistence of OFDMbased systems beyond $3 \mathrm{G}$ with Fixed Service Microwave Systems", Journal of Communications and Networks, vol. 8, no. 2, (2006).

[15] ITU-R Rec. SM.1448, Determination of the coordination area around an earth station in the frequency bands between $100 \mathrm{MHz}$ and $105 \mathrm{GHz},(\mathbf{2 0 0 0})$.

[16] ITU-R Rec. S.1432, Apportionment of the allowable error performance degradations to Fixed-Satellite Service (FSS) hypothetical reference digital paths arising from time invariant interference for syste ms operating below $15 \mathrm{GHz}$, (2000). 


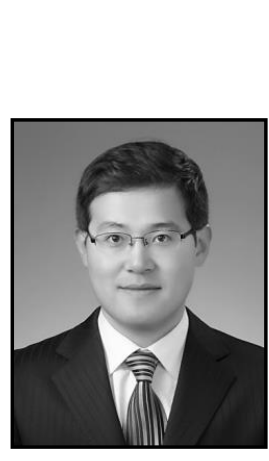

\section{Author}

Han-Shin Jo is an Assistant Professor with the Department of Electronics and Control Engineering, Hanbat National University in Korea. He was a Postdoctoral Research Fellow in Wireless Networking and Communications Group, the Department of Electrical and Computer Engineering, the University of Texas at Austin from 2009-11. Dr. Jo developed LTE systems in Samsung Eletronics in 2011-12. He received the B.S., M.S., and Ph.D. degrees in Electrical and Electronics Engineering from Yonsei University Seoul, Korea, in 2001, 2004, and 2009, respectively. He received 2011 ETRI Journal Award. His research interests include Small cells, Heterogeneous network, Wireless ad-hoc network, Stochastic geometry, and Wireless broadband transmission. 
International Journal of Multimedia and Ubiquitous Engineering Vol. 10, No. 1 (2015) 